\title{
High-resolution core-level spectroscopy study of the ultrathin aluminum oxide film on $\mathrm{NiAl}(110)$
}

\author{
N. M. Martin, J. Knudsen, S. Blomberg, J. Gustafson, J. N. Andersen, and E. Lundgren \\ Division of Synchrotron Radiation Research, Lund University, Box 118, SE-22100, Sweden \\ H. Härelind Ingelsten, P.-A. Carlsson, and M. Skoglundh \\ Competence Center for Catalysis, Chalmers University of Technology, SE-41296, Göteborg, Sweden \\ A. Stierle \\ Arbeitsgruppe Festkörperphysik, Grenzflächen Fachbereich 7 Physik, Universität Siegen Walter-Flex-Str. 3, D-57072 Siegen, Germany \\ Max-Planck Institut für Metallforschung, Heisenbergstr. 3, D-70569 Stuttgart, Germany \\ G. Kresse \\ Institut für Materialphysik, Universität Wien, A-1090 Wien, Austria
}

(Received 26 November 2010; published 22 March 2011)

\begin{abstract}
We have studied the ultrathin aluminum oxide film on $\mathrm{NiAl}(110)$ by a combination of high-resolution core-level spectroscopy and density functional theory calculations. Energy-dependent core-level data from the O $1 s$ and Al $2 p$ levels allows for a distinction between oxygen and aluminum atoms residing at the surface or inside the aluminum oxide film. A comparison to calculated core-level binding energies from the recent model by Kresse et al. [Science 308, 1440 (2005)] reveals good agreement with experiment, and the complex spectroscopic signature of the thin $\mathrm{Al}$ oxide on $\mathrm{NiAl}(110)$ can be explained. Our assignment of a shifted component in the $\mathrm{O}$ $1 s$ spectra to oxygen atoms at the surface with a particular $\mathrm{Al}$ and oxygen coordination may have implications for the interpretation of photoelectron-diffraction experiments from similar ultrathin aluminum oxide films.
\end{abstract}

DOI: 10.1103/PhysRevB.83.125417

PACS number(s): 68.47.Gh, 79.60.Dp, 71.15.Mb

\section{INTRODUCTION}

Well-ordered oxide films grown on single-crystal substrates have attracted considerable attention in the past 20 years mainly because of various applications in microelectronics, catalysis, anticorrosion, nanotemplates, coatings, and sensors. ${ }^{1-7}$ One of the most common model substrates for model catalysts is a thin aluminum oxide film grown on $\mathrm{NiAl}(110)$. It has previously been shown that well-ordered ultrathin oxide layers of about $0.5 \mathrm{~nm}$ thickness may be grown by oxidation of $\mathrm{NiAl}(110) \cdot{ }^{8-14}$ The high melting temperature of the alloy facilitates preparation temperatures sufficiently high to allow ordering of the oxide. Since its discovery, significant efforts have been made to solve the atomic structure of the oxide layer. ${ }^{9-11,15-19}$ Despite the fact that the oxide has been intensively studied, its detailed structure remained unknown until recently. ${ }^{1,11}$ In this contribution we present a new high-resolution core-level spectroscopy (HRCLS) study of the aluminum oxide film grown on $\mathrm{NiAl}(110)$, which extends previous X-ray photoelectron spectroscopy studies. ${ }^{9,20-22}$ Using energy-dependent core-level measurements for nondestructive depth profiling we are able to assign the different experimental $\mathrm{O} 1 s$ and $\mathrm{Al} 2 p$ core-level shifts unambiguously to surface and interface atoms, respectively. Calculated binding energies using the model by Kresse ${ }^{1}$ are in good agreement with our experimental findings. The present HRCLS study therefore supports the model by Kresse et al. and allows for a correct assignment of the different HRCL components to specific atomic sites. Most surprisingly, a component in the $\mathrm{O}$ $1 s$ binding-energy range which previously has been assigned to the full topmost oxygen layer of the oxide film ${ }^{22}$ is shown to originate from only $30 \%$ of the surface oxygen atoms.

\section{EXPERIMENT}

The HRCLS experiments were carried out at Beamline $\mathrm{I} 311^{23}$ at the Swedish synchrotron facility MAX-lab in Lund, Sweden. For the experiments a normal emission angle was used and the $\mathrm{O} 1 s$ level and Al $2 p$ level were recorded with photon energies between $620-900 \mathrm{eV}$ and $105-400 \mathrm{eV}$, respectively.

The sample was mounted on a tungsten wire through which it could be heated by applying an electric current and the temperature was measured by a chromel-alumel thermocouple spot welded on the side of the crystal. The $\mathrm{NiAl}(110)$ crystal was cleaned as described in Ref. 24. All measurements were performed at $100 \mathrm{~K}$. A Fermi level was recorded after each spectra and used as binding-energy reference for calibration. The thin aluminum oxide film was prepared as described in Refs. 9-11 and the surface ordering was checked by lowenergy electron diffraction (LEED), ${ }^{9,10}$

The decomposition procedure followed that in Ref. 24. Starting with the $\mathrm{Al} 2 p$ level, the four decomposed peaks each consist of two components due to the spin-orbit coupling of the $\mathrm{Al} 2 p_{1 / 2}$ and $\mathrm{Al} 2 p_{3 / 2}$ levels. The intensity of the $\mathrm{Al} 2 p_{1 / 2}$ was set to be half the intensity of the $\mathrm{Al} 2 p_{3 / 2}$ component. The full width at half maximum (FWHM) and asymmetry was found to be the same for the bulk and the interface components, while the $\mathrm{Al}_{i}$ and $\mathrm{Al}_{s}$ (see below) emission displayed an increased FWHM due to a large number of $\mathrm{Al}$ atoms with different chemical surroundings introducing small binding-energy shifts. The asymmetry parameter for the $\mathrm{Al}$ atoms from the oxide ranged between 0.02 and 0.05 . For the $\mathrm{O} 1 \mathrm{~s}$ we found a FWHM of $1.2 \mathrm{eV}$ for a photon energy of $900 \mathrm{eV}$ which was found to decrease with decreasing photon 
energy due to the improved experimental resolution at lower photon energies. The asymmetry ratio was found to be 0.07 .

The density functional theory (DFT) calculations were performed by using the Vienna $a b$ initio simulation package (VASP) ${ }^{25,26}$ employing the projector augmented wave method $^{27}$ with a cut-off energy of $250 \mathrm{eV}$ and PW91 gradient corrections. ${ }^{28}$ The most favorable model was determined by extensive modeling with simulated annealing and as well with atomically resolved scanning tunneling images. ${ }^{1}$

The surface core-level shifts (SCLS) were calculated (including final-state contributions) as the difference between the core-level binding energies of the surface and the bulk ${ }^{29}$ with the core-level binding energy given by $E_{\mathrm{CL}}=E\left(n_{c}-\right.$ $1)-E\left(n_{c}\right)$, where $E\left(n_{c}-1\right)$ is the total energy of the excited atom after the removal of one electron from the core and $E\left(n_{c}\right)$ is the energy for the unexcited ground state.

\section{RESULTS AND DISCUSSION}

According to several previous studies and the model by Kresse et $a l .{ }^{1}$ the ultrathin aluminum oxide film grown on $\mathrm{NiAl}(110)$ consists of a double oxide layer with $\mathrm{NiAl}-\mathrm{Al}_{i}{ }^{-}$ $\mathrm{O}_{i}-\mathrm{Al}_{s}-\mathrm{O}_{s}$ stacking. The different layers of the model ${ }^{1}$ are presented in Fig. 1. At the interface the number of $\mathrm{Al}$ atoms is identical to the number of $\mathrm{NiAl}$ unit cells, with a strong preference for the $\mathrm{Al}$ atoms to be located above the Ni rows. In more detail, the four different layers consist of an interfacial $\mathrm{Al}$ layer $\mathrm{Al}_{i}$ [Fig. 1(a)]; an interfacial oxygen layer $\mathrm{O}_{i}$ [Fig. 1(b)];

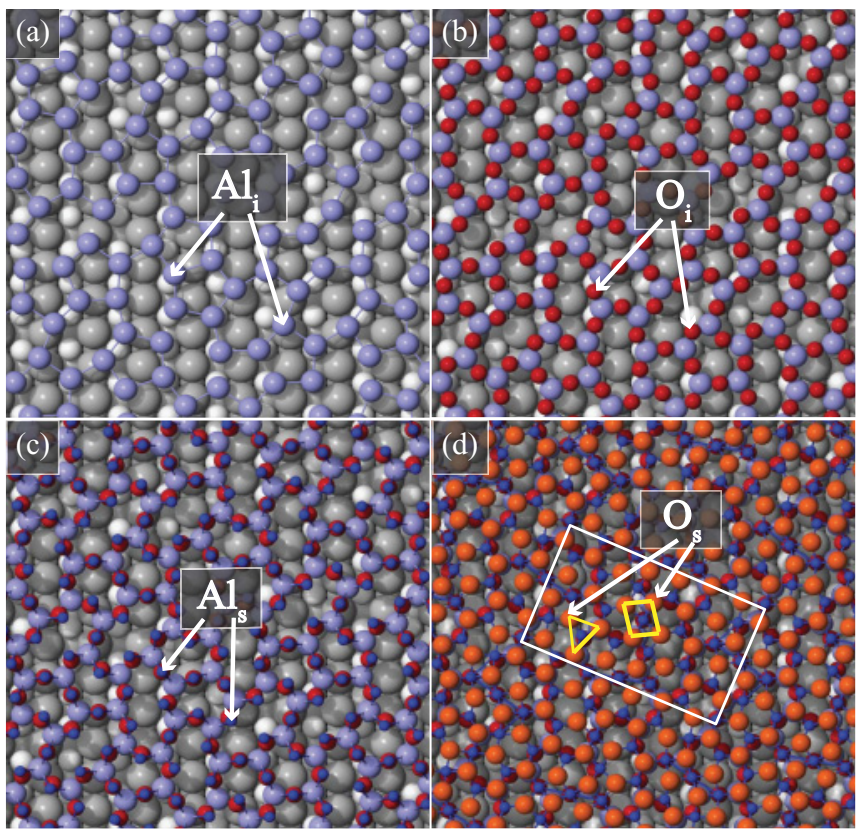

FIG. 1. (Color online) Structural model of the ultrathin aluminum oxide film grown on $\mathrm{NiAl}(110)$. (a) The interface $\mathrm{Al}$ atoms $\left(\mathrm{Al}_{i}\right.$, light blue). The gray and white atoms represent the $\mathrm{Al}$ and $\mathrm{Ni}$ atoms in the the $\mathrm{NiAl}(110)$ substrate, respectively. (b) The interface oxygen atoms $\left(\mathrm{O}_{i}\right.$, red). (c) The surface $\mathrm{Al}$ atoms $\left(\mathrm{Al}_{s}\right.$, blue). The $\mathrm{Al}_{s}$ atoms are situated on top of the $\mathrm{O}_{i}$ atoms below. (d) The surface oxygen atoms $\left(\mathrm{O}_{s}\right.$, orange). The unit cell of the oxide structure is indicated, as well as the pyramid and tetrahedra structural units within the unit cell. a second surface layer of $\mathrm{Al}$ atoms $\mathrm{Al}_{s}$ [Fig. 1(c)] directly above the $\mathrm{O}_{i}$ atoms; and, finally, the surface oxygen layer, $\mathrm{O}_{s}$ [Fig. 1(d)]

In the model by Kresse et al., ${ }^{1}$ the stoichiometry of the film was determined to be $\mathrm{Al}_{10} \mathrm{O}_{13}$ and not $\mathrm{Al}_{2} \mathrm{O}_{3}$ as it was previously believed. The $\mathrm{Al}_{i}$ atoms are arranged in pentagon-heptagon pairs above the $\mathrm{Ni}$ atoms because of the preferred chemical short-range order: $\mathrm{Al}_{i}$ atoms prefer to bind to $\mathrm{Ni}$ surface atoms. Each $\mathrm{O}_{i}$ atom binds to two $\mathrm{Al}_{i}$ atoms below and one $\mathrm{Al}_{s}$ atom above. Based on STM measurements it was found that the $\mathrm{Al}_{s}$ atoms bind to additionally three or four $\mathrm{O}_{s}$ atoms, forming pyramids or tetrahedra with the $\mathrm{O}_{i}$ and $\mathrm{O}_{s}$ atoms, as indicated in Fig. 1(d). At the surface, the $\mathrm{O}_{s}$ atoms are arranged in squares (due to the pyramids) and triangles (due to the tetrahedras) and each $\mathrm{O}_{s}$ atom binds to three $\mathrm{Al}_{s}$ similar to the arrangement of the atoms at the surface of an ordered $\theta$ $\mathrm{Al}_{2} \mathrm{O}_{3}$ layer on $\mathrm{NiAl}(100) .{ }^{17}$ This arrangement differs slightly from the octahedrally and tetrahedrally coordinated $\mathrm{Al}_{s}$ atoms reported earlier in Refs. 30 and 31. Below we will show how this latter surface oxygen arrangement is in line with our HRCLS measurements.

In Fig. 2(a) we show the $\mathrm{Al} 2 p$ and $\mathrm{O} 1 s$ core levels recorded at normal emission with photon energies of 130 and $900 \mathrm{eV}$, respectively, from the ultrathin aluminum oxide on $\mathrm{NiAl}(110)$. The calculated binding energies from the model in Ref. 1 are indicated as black solid spheres. A comparison between the calculated and experimentally observed core-level shifts is given in Table I. It is immediately clear that the broad appearance of the core-level spectra related to the oxidized $\mathrm{Al}$ atoms is due to a large number of shifted components originating from $\mathrm{Al}$ atoms in the oxide film with slightly different local environments.

We will start to discuss the core-level emission from the Al $2 p$ level and discuss each component in terms of relative shifts from the bulk emission (light blue) at a binding energy of $72.5 \mathrm{eV}$. The $\mathrm{Al} 2 p$ recorded with a photon energy of $130 \mathrm{eV}$ is presented in Fig. 2(a, right). The spectrum can be decomposed into four components as shown in Fig. 2(a), each of them split by $0.4 \mathrm{eV}$ and with a ratio of $1 / 2$.

TABLE I. Comparison between the calculated (Calc) and experimentally (Exp) observed core-level shifts (CLS) for the ultrathin oxide $\mathrm{Al}_{10} \mathrm{O}_{13}$ on $\mathrm{NiAl}(110)$. The core-level shifts are referred to the binding energy of the bulk $\mathrm{Al} 2 p$ [Al atoms located in the bulk of the $\mathrm{NiAl}(110)$ crystal] and the average core-level binding energy of the surface oxygen atoms with low binding energy [O $1 s \mathrm{O}_{i}$ and $\left.\mathrm{O} 1 s \mathrm{O}_{s}(1)\right] . \mathrm{O} 1 s \mathrm{O}_{s}(2)$ are those oxygen atoms at the surface layer that have an $\mathrm{Al}$ atom underneath and do not reside very close to another oxygen atom, while $\mathrm{O} 1 s \mathrm{O}_{s}(1)$ are the remaining atoms at the surface.

\begin{tabular}{lcc}
\hline \hline Atom & Exp CLS $(\mathrm{eV})$ & Calc CLS $(\mathrm{eV})$ \\
\hline $\mathrm{Al} 2 p_{3 / 2}$ bulk & 0.00 & 0.00 \\
$\mathrm{Al} 2 p_{3 / 2} \mathrm{I}$ & -0.10 & $-0.60--0.30$ \\
$\mathrm{Al} 2 p_{3 / 2} \mathrm{Al}_{i}$ & 1.00 & $1.00-1.40$ \\
$\mathrm{Al} 2 p_{3 / 2} \mathrm{Al}_{s}$ & 2.27 & $1.71-2.67$ \\
$\mathrm{O} 1 s \mathrm{O}_{i}$ & 0.00 & $-0.41-0.54$ \\
$\mathrm{O} 1 s \mathrm{O}_{s}(1)$ & 0.00 & $-0.41-0.54$ \\
$\mathrm{O} 1 s \mathrm{O}_{s}(2)$ & 1.23 & $1.05-1.14$ \\
\hline \hline
\end{tabular}



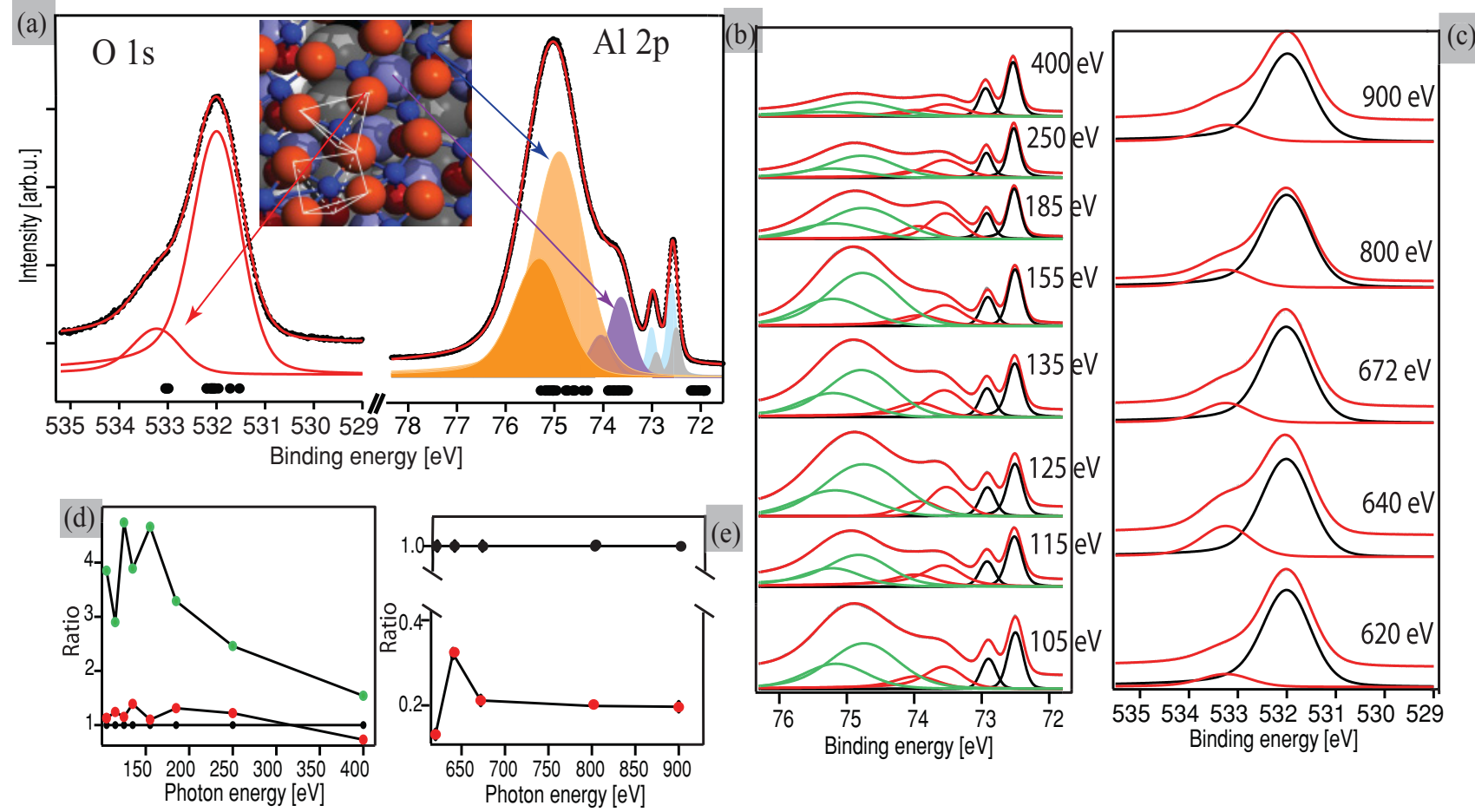

FIG. 2. (Color online) High-resolution core-level spectra from the ultrathin aluminum oxide on $\mathrm{NiAl}(110)$. (a) Al $2 p$ spectrum (right) recorded at $130 \mathrm{eV}$. Four components are clearly visible, each with a $0.4-\mathrm{eV}$ spin-orbit split with the ratio of $1 / 2$. The components can be identified as $\mathrm{Al}$ in the $\mathrm{NiAl}$ bulk (blue), $\mathrm{Al}$ at the interface between the $\mathrm{Al}$ oxide film and the $\mathrm{NiAl}(110)$ substrate (gray), $\mathrm{Al}_{\text {in }}$ the $\mathrm{Al}_{i}$ layer (violet), and $\mathrm{Al}$ in the $\mathrm{Al}_{s}$ layer (orange). $\mathrm{O} 1 s$ spectrum (left) recorded at $900 \mathrm{eV}$. Two components can be identified with the ratio of 0.19 ; see text. The more intense component originates from the $\mathrm{O}$ atoms in the $\mathrm{O}_{i}$ layer and the $\mathrm{O}$ atoms in the surface layer which reside very close to another $\mathrm{O}_{s}$ atom. The weaker component is due to $\mathrm{O}$ atoms in the $\mathrm{O}_{s}$ layer which have the $\mathrm{Al}_{i}$ atoms underneath and do not reside very closely to another oxygen surface atom (see inset and text). The calculated core-level binding energies are indicated by solid spheres. Energy-dependent measurements from (b) the $\mathrm{Al} 2 p$ level and (c) the $\mathrm{O} 1 s$ level. The integrated area from (d) the $\mathrm{Al} 2 p$ components and (e) the O $1 s$ components.

The component at the lowest binding energy $(72.4 \mathrm{eV}$, gray) is the so-called interface component, which is electron emission from the $\mathrm{Al}$ atoms in the $\mathrm{NiAl}(110)$ surface directly below the ultrathin $\mathrm{Al}$ oxide. These $\mathrm{Al}$ atoms are shown in Fig. 1(a) as dark gray atoms and in Table I they are denoted as $\mathrm{Al} 2 p_{3 / 2}$ I. The shift with respect to the $\mathrm{Al} 2 p$ from the $\mathrm{Al}$ atoms in the $\mathrm{NiAl}(110)$ bulk is found to be $-0.1 \mathrm{eV}$. Similar interface shifts have been observed for other ultrathin oxides and metal systems. ${ }^{32-35}$ This component has not been observed previously for the present system, but a similar component can be observed in the case of thin oxides on $\mathrm{Al}(100) .{ }^{36}$ Although the agreement between the calculated and experimental binding energies is not perfect, the calculated binding energies shown in Fig. 3 and Table I confirm a shift toward lower binding energies for these $\mathrm{Al}$ atoms.

The two $\mathrm{Al} 2 p$ components shifted 1.00 and $2.27 \mathrm{eV}$ are assigned to photoemission from $\mathrm{Al}$ atoms in the $\mathrm{Al}_{10} \mathrm{O}_{13}$ film. To distinguish the photoemission from $\mathrm{Al}_{i}$ and $\mathrm{Al}_{s}$ atoms we used energy-dependent photoemission measurements, as shown in Fig. 2(b) and 2(d). It can be seen that, at higher energies, the $2.27 \mathrm{eV}$ shifted component decreases more rapidly in intensity as compared to the $1.00 \mathrm{eV}$ shifted component which is also decreasing but not so fast. The measurements reveal that the $1.00 \mathrm{eV}$ shifted component should be assigned to $\mathrm{Al}$ atoms located at a depth intermediate between the $\mathrm{Al}$ atoms in the $\mathrm{NiAl}(110)$ substrate and the $\mathrm{Al}$ atoms from the emission found at $2.27 \mathrm{eV}$. Thus the $1.00 \mathrm{eV}$ shifted component originates from the $\mathrm{Al}_{i}$ atoms and the $2.27 \mathrm{eV}$ component originates from the $\mathrm{Al}_{s}$ atoms. This agrees well with previous HRCLS studies. ${ }^{9,22,37}$ Furthermore, the calculated binding energies shown in Fig. 2(a) as black spheres are in excellent agreement with the experimentally observed shifts.

The $\mathrm{O} 1 s$ spectrum recorded with photon energy of $900 \mathrm{eV}$ is presented in Fig. 2(a) left. It can be seen that the emission is broad and asymmetric and exhibits a shoulder on the highbinding-energy side. The spectrum can be decomposed into two components. The ratio between the two components using a photon energy of $900 \mathrm{eV}$ is found to be 0.19 and should, at this high kinetic electron energy, reflect the real stoichiometry unaffected by diffraction effects. The shift between the two components is found to be $1.23 \mathrm{eV}$. Previously, the higherbinding-energy component was assigned to a $\mathrm{O}^{2-}$ surface species, ${ }^{22}$ and in the case of the similar ultrathin aluminum oxide on $\mathrm{NiAl}(111)$ a similar component was assigned to the $\mathrm{O}_{s}$ layer. $^{38}$

The energy-dependent measurements shown in Figs. 2(c) and 2(e) reveal strong diffraction effects at lower kinetic energies; however, at higher energies, the smaller component 


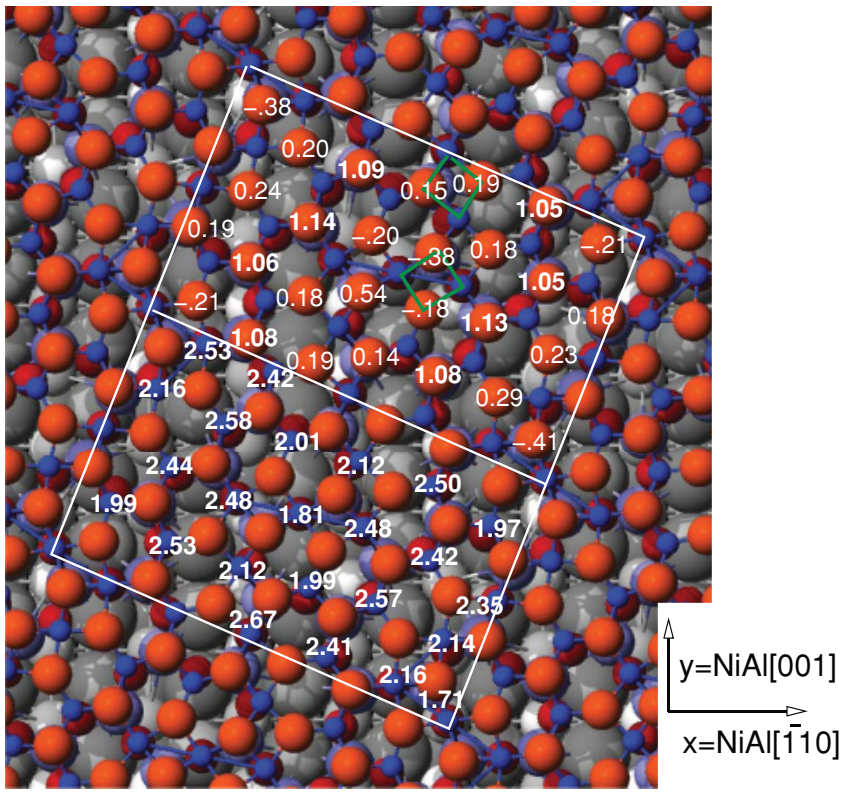

FIG. 3. (Color online) Calculated core-level shifts for the Al $2 p$ (blue, bottom rectangle) and $\mathrm{O} 1 s$ (orange, top rectangle) atoms in the $\mathrm{O}_{s}$ and $\mathrm{Al}_{s}$ surface layer of the $\mathrm{Al}_{10} \mathrm{O}_{13}$ oxide film. The $\mathrm{Al} 2 p$ shifts are calculated with respect to the $\mathrm{Al}$ bulk binding energy, while the $\mathrm{O} 1 s$ shift is presented with respect to the average oxygen shift of the surface oxygen atoms $\mathrm{O}_{s}$ with low binding energy.

decreases more rapidly in intensity as compared to the larger component, suggesting that the small component is indeed from the atoms in the $\mathrm{O}_{s}$ layer. However, it is clear that not all of the oxygen atoms in the $\mathrm{O}_{s}$ layer can contribute to the intensity, since a significant higher intensity would in that case be expected.

In order to elucidate the nature of the weak $1.23 \mathrm{eV}$ shifted $\mathrm{O} 1 s$ component, we present the calculated binding-energy shifts for all $\mathrm{O}_{s}$ and $\mathrm{Al}_{s}$ in Fig. 3. Of the 28 surface $\mathrm{O}_{s}$ atoms, 8 show a shift of about $1.1 \mathrm{eV}$ compared to the other $\mathrm{O}_{s}$ atoms. This compares well with the experimentally observed shift of $1.23 \mathrm{eV}$. The oxygen atoms with a large shift always have an $\mathrm{Al}_{i}$ atom directly underneath, making the electrostatic field at these sites particularly attractive. A few oxygen atoms with $\mathrm{Al}_{i}$ atoms underneath do not show the strong binding-energy shift, but those oxygen atoms always reside very close to another oxygen surface atom forming an O-Al-O-Al square on the surface (marked dark green in Fig. 3). The close vicinity of the second negative oxygen ion obviously counterbalances the attractive field of the interfacial $\mathrm{Al}_{i}$ atom.

The ratio between the $\mathrm{O}_{s}$ atoms with a strong shift and the overall oxygen atoms in the unit cell is calculated to be 0.18 , which should be compared to the experimental value of 0.19 , giving us further confidence in our assignment. From our analysis, it is therefore clear that the weak component in the $\mathrm{O} 1 s$ spectra originates from a fraction of the $\mathrm{O}_{s}$ atoms while the more intense components have contributions from oxygen in the $\mathrm{O}_{s}$ layer as well as from the $\mathrm{O}_{i}$ layer. A comparison between the calculated and experimentally core-level shifts is shown in Table I.
In the case of the ultrathin aluminum oxide film on $\operatorname{NiAl}(111)^{6}$ the structural units forming that oxide are the same as in the present case. In a recent photoelectron diffraction study of the $\mathrm{NiAl}(111)$ aluminum oxide system, ${ }^{38}$ the highbinding-energy component in the $\mathrm{O} 1 s$ level was assigned to all the oxygen atoms in the topmost surface layer (designated $\mathrm{O}_{t}$ layer in Ref. 38). Comparing the intensity and the bindingenergy shift of the high-binding-energy component in the present study with that in Ref. 38, the agreement is excellent. Since the structural building blocks are the same in both oxides, it is likely that also in the case of the ultrathin oxide on $\mathrm{NiAl}(111)$ the high-binding-energy $\mathrm{O} 1 s$ component originates from the oxygen atoms which have $\mathrm{Al}_{i}$ atoms directly underneath and which do not reside close to another oxygen surface atom. In the present case, this amounts to approximately $30 \%$ of the oxygen atoms in the $\mathrm{O}_{s}$ layer ( 8 of 28). If the assignment is the same in the case of the ultrathin aluminum oxide on $\mathrm{NiAl}(111)$, it may have implications for the interpretation of the electron-diffraction measurements from the $\mathrm{O} 1 s$ level in Ref. 38.

Finally, a careful inspection of the bottom rectangle in Fig. 3 shows that the Al atoms with three- and fourfold coordination to oxygen span shifts of $1.99-2.58 \mathrm{eV}$ and $1.71-2.67 \mathrm{eV}$, respectively. Figure 3 therefore reveals that there is no clear distinction between the three- and fourfold oxygen coordinated $\mathrm{Al}$ atoms in terms of their binding-energy shifts, correcting previous assignments of the $\mathrm{Al} 2 p$ core-level shifts. ${ }^{20}$

\section{SUMMARY}

In summary, we have studied the aluminum oxide grown on the $\mathrm{NiAl}(110)$ substrate by the use of HRCLS and DFT calculations. In agreement with several other HRCLS studies, the presented spectra reveal a number of components which belong to species in different layers in the structure. A detailed comparison between experimental and computed core-level shifts using the structural model by Kresse et al. ${ }^{1}$ makes it possible to assign experimental binding energies to certain atoms in the structure. The agreement between experimental and calculated data is excellent, thus HRCLS from the structure is in line with the model in Ref. 1. In particular, a shifted component in the $\mathrm{O} 1 s$ spectrum, which has previously been assigned to all the surface oxygen atoms, can be assigned to surface oxygen atoms which do not reside very close to another oxygen atom corresponding to only $30 \%$ of the surface oxygen atoms. This assignment may have consequences for the interpretation of photoelectron-diffraction data.

\section{ACKNOWLEDGMENTS}

This work was financially supported by the foundation for strategic research (SSF), the Swedish Research Council, the Crafoord Foundation, the Knut and Alice Wallenberg Foundation, the Anna and Edwin Berger Foundation, the Danish Council for Independent Research, and the EC ARI grant. The MAX-lab staff are gratefully acknowledged. 
${ }^{1}$ G. Kresse, M. Schmid, E. Napetschnig, M. Shishkin, L. Köhler, and P. Varga, Science 308, 1440 (2005).

${ }^{2}$ C. T. Campbell, Surf. Sci. Rep. 27, 1 (1997).

${ }^{3}$ C. R. Henry, Surf. Sci. Rep. 31, 231 (1998).

${ }^{4}$ H. Iwasaki and K. Sudoh, Jpn. J. Appl. Phys. 41, 7496 (2002).

${ }^{5}$ E. Lundgren, A. Mikkelsen, J. N. Andersen, G. Kresse, M. Schmid, and P. Varga, J. Phys. Condens. Matter 18, R481 (2006).

${ }^{6}$ M. Schmid, G. Kresse, A. Buchsbaum, E. Napetschnig, S. Gritschneder, M. Reichling, and P. Varga, Phys. Rev. Lett. 99, 196104 (2007).

${ }^{7}$ H.-J. Freund and G. Pacchioni, Chem. Soc. Rev. 37, 2224 (2008).

${ }^{8}$ H. Isern and G. R. Castro, Surf. Sci. 211/212, 865 (1989).

${ }^{9}$ R. M. Jaeger, H. Kuhlenbeck, H.-J. Freund, M. Wuttig, W. Hofmann, R. Franchy, and H. Ibach, Surf. Sci. 259, 235 (1991).

${ }^{10}$ J. Libuda, F. Winkelmann, M. Bäumer, H. J. Freund, T. Bertams, H. Neddermeyer, and K. Müller, Surf. Sci. 318, 61 (1994).

${ }^{11}$ A. Stierle, F. Renner, R. Streitel, H. Dosch, W. Drube, and B. C. Cowie, Science 303, 1652 (2004).

${ }^{12}$ M. Kulawik, N. Nilius, H-P. Rust, and H. -J. Freund, Phys. Rev. Lett. 91, 256101 (2003).

${ }^{13}$ N. Nilius, M. Kulawik, H. -P. Rust, and H. -J. Freund, Phys. Rev. B 69, 121401(R) (2004).

${ }^{14}$ M. Schmid, M. Shishkin, G. Kresse, E. Napetschnig, P. Varga, M. Kulawik, N. Nilius, H.-P. Rust, and H.-J. Freund, Phys. Rev. Lett. 97, 046101 (2006).

${ }^{15}$ Th. Bertrams, A. Brodde, and H. Neddermeyer, J. Vac. Sci. Technol. B 12, 2122 (1994).

${ }^{16}$ A. Stierle, F. Renner, R. Streitel, and H. Dosch, Phys. Rev. B 64, 165413 (2001).

${ }^{17}$ A. Stierle, V. Formoso, F. Comin, and R. Franchy, Surf. Sci. 467, 85 (2000).

${ }^{18}$ M. Finnis, A. Lozovoi, and A. Alavi, Annu. Rev. Mater. Res. 35, 167 (2005).

${ }^{19}$ K. Müller, H. Lindner, D. M. Zehner, and G. Ownby, Verh. Dtsch. Phys. Ges. 25, 1130 (1990).
${ }^{20}$ A. Mulligan, V. Dhanak, and M. Kadodwala, Langmuir 21, 8312 (2005).

${ }^{21}$ M. Bäumer and H.-J. Freund, Prog. Surf. Sci. 61, 127 (1999).

${ }^{22}$ J. Liduda, M. Frank, A. Sandell, S. Andersson, P. A. Bruhwiler, M. Baumer, N. Mårtensson, and H.-J. Freund, Surf. Sci. 384, 106 (1997).

${ }^{23}$ R. Nyholm, J. N. Anderssen, U. Johansson, B. Jensen, and I. Lindau, Nucl. Instrum. Methods A 467, 520 (2001).

${ }^{24}$ A. Stierle, C. Tieg, H. Dosch, V. Formoso, E. Lundgren, J. N. Andersen, L. Köhler, and G. Kresse, Surf. Sci. 529, L263 (2003).

${ }^{25}$ G. Kresse and J. Hafner, Phys. Rev. B 47, 558 (1993).

${ }^{26}$ G. Kresse and J. Furthmuller, Comput. Mater. Sci. 6, 15 (1996).

${ }^{27}$ G. Kresse and D. Joubert, Phys. Rev. B 59, 1758 (1999).

${ }^{28}$ J. P. Perdew, J. A. Chevary, S. H. Vosko, K. A. Jackson, M. R. Pederson, D. J. Singh, and C. Fiolhais, Phys. Rev. B 46, 6671 (1992).

${ }^{29}$ L. Köhler and G. Kresse, Phys. Rev. B 70, 165405 (2004).

${ }^{30}$ D. R. Jennison and A. A. Bogicevic, Surf. Sci. 464, 108 (2000).

${ }^{31}$ X. G. Wang, A. Chaka, and M. Scheffler, Phys. Rev. Lett. 84, 3650 (2000).

${ }^{32}$ M. Todorova, Surf. Sci. 541, 101 (2003).

${ }^{33}$ P. Kostelnik, N. Seriani, G. Kresse, A. Mikkelsen, E. Lundgren, V. Blum, T. Sikola, P. Varga, and M. Schmid, Surf. Sci. 601, 1574 (2007).

${ }^{34}$ J. Gustafson, Phys. Rev. B 71, 115442 (2005).

${ }^{35}$ A. Stierle, New J. Phys. 9, 331 (2007)

${ }^{36}$ C. Berg, S. Raaen, A. Borg, J. N. Andersen, E. Lundgren, and R. Nyholm, Phys. Rev. B 47, 13063 (1993).

${ }^{37}$ A. Sandell, J. Libuda, P. Bruhwiler, S. Andersson, A. Maxwell, M. Bäumer, N. Mårtensson, and H.-J. Freund, J. Electron Spectrosc. Relat. Phenom. 76, 301 (1995)

${ }^{38}$ E. Vesselli, A. Baraldi, S. Lizzit, and G. Comelli, Phys. Rev. Lett. 105, 046102 (2010). 